\title{
Quantification of the physical and physiological load of a boxing-specific simulation protocol
}

\author{
Edward Thomson ${ }^{1}$ and Kevin Lamb ${ }^{1}$ \\ ${ }^{1}$ Faculty of Medicine, Dentistry and Life Sciences, Department of Sport and Exercise \\ Sciences, University of Chester, Chester Campus, Parkgate Road, Chester, Cheshire, CH1 \\ 4BJ.
}

\begin{abstract}
The aim of the study was to determine the physical and physiological responses to simulated amateur boxing of $3 \times 3$-minute rounds. Using an externally valid technical and ambulatory demand, 28 amateur boxers (mean \pm SD; age $22.4 \pm 3.5$ years, body mass $67.7 \pm 10.1 \mathrm{~kg}$, stature $171 \pm 9 \mathrm{~cm}$ ) completed the protocol following familiarisation. The physiological load was determined continuously via collection of mean $\left(\mathrm{HR}_{\text {mean }}\right)$ and peak $\left(\mathrm{HR}_{\text {peak }}\right)$ heart rate, breath-by-breath oxygen uptake ( $\left.\dot{V}^{\prime}{ }_{2}\right)$, aerobic energy expenditure $\left(E_{\mathrm{aar}}\right)$, excess carbon dioxide production $\left(\mathrm{CO}_{\text {2excess }}\right)$, ratings of perceived exertion (RPE) and postperformance blood lactate. Physical performance was quantified as the acceleration delivered to the target by punches. $\mathrm{HR}_{\text {mean }}$ and $\mathrm{HR}_{\text {peak }}$ were found to exceed 165 and $178 \mathrm{~b} \cdot \mathrm{min}^{-1}$, absolute $V O_{2}>124.6 \mathrm{ml} \cdot \mathrm{kg}^{-1}, \mathrm{EE}_{\text {aer }}>30.7$ $\mathrm{kcal} \cdot \mathrm{min}^{-1}$ and acceleration via 78 punches $>2697 \mathrm{~g}$ during each round. Mean blood lactate $\left(4.6 \mathrm{mmol} \cdot \mathrm{l}^{-1}\right)$ and $\mathrm{CO}_{\text {2excess }}\left(438.7 \mathrm{ml} \cdot \mathrm{min}^{-1}\right)$ were higher than typical resting values reflecting a notable anaerobic contribution. RPEs reinforced the intensity of exercise was strenuous ( $>6-8)$. For all measures, there were typically increases $(P<0.05$; moderate $E S)$ across rounds. Accordingly, boxers might consider high-intensity ( $>90 \% \quad \dot{V} \quad \mathrm{O}_{2 \max }$ ) interval training in anticipation such exercise yields improvements in aerobic conditioning. Moreover, the current simulation protocol - the boxing conditioning and fitness test (BOXFIT) - could be used as a form of training per se and as a means to monitor intervention-based changes in aspects of boxing-related physiology and performance.
\end{abstract}

Keywords: Combat sports, boxing simulation, validity 


\title{
Quantification of the physical and physiological load of a boxing-specific simulation protocol
}

\begin{abstract}
The aim of the study was to determine the physical and physiological responses to simulated amateur boxing of $3 \times 3$-minute rounds. Using an externally valid technical and ambulatory demand, 28 amateur boxers (mean \pm SD; age $22.4 \pm 3.5$ years, body mass $67.7 \pm 10.1 \mathrm{~kg}$, stature $171 \pm 9 \mathrm{~cm}$ ) completed the protocol following familiarisation. The physiological load was determined continuously via collection of mean $\left(\mathrm{HR}_{\text {mean }}\right)$ and peak $\left(\mathrm{HR}_{\text {peak }}\right)$ heart rate, breath-by-breath oxygen uptake ( $V^{\prime} \mathrm{O}_{2}$ ), aerobic energy expenditure (EEar), excess carbon dioxide production $\left(\mathrm{CO}_{\text {2excess }}\right)$, ratings of perceived exertion (RPE) and postperformance blood lactate. Physical performance was quantified as the acceleration delivered to the target by punches. $\mathrm{HR}_{\text {mean }}$ and $\mathrm{HR}_{\text {peak }}$ were found to exceed 165 and $178 \mathrm{~b} \cdot \mathrm{min}^{-1}$, absolute $\dot{V} \mathrm{O}_{2}>124.6 \mathrm{ml} \cdot \mathrm{kg}^{-1}, \mathrm{EE}_{\text {aer }}>30.7$ $\mathrm{kcal} \cdot \mathrm{min}^{-1}$ and acceleration via 78 punches $>2697 \mathrm{~g}$ during each round. Mean blood lactate $\left(4.6 \mathrm{mmol} \cdot \mathrm{l}^{-1}\right)$ and $\mathrm{CO}_{\text {2excess }}\left(438.7 \mathrm{ml} \cdot \mathrm{min}^{-1}\right)$ were higher than typical resting values reflecting a notable anaerobic contribution. RPEs reinforced the intensity of exercise was strenuous ( $>6-8)$. For all measures, there were typically increases $(P<0.05$; moderate $E S)$ across rounds. Accordingly, boxers might consider high-intensity ( $\left.>90 \% \quad \dot{V} \mathrm{O}_{2 \max }\right)$ interval training in anticipation such exercise yields improvements in aerobic conditioning. Moreover, the current simulation protocol - the boxing conditioning and fitness test (BOXFIT) - could be used as a form of training per se and as a means to monitor intervention-based changes in aspects of boxing-related physiology and performance.
\end{abstract}

Keywords: Combat sports, boxing simulation, validity 


\section{Introduction}

The quantification of the internal (physiological) and external (movement) demands of competitive athletic performance for guiding training is an important endeavour in sports science (Bishop et al., 2008). However, the collection and assessment of actual sports performance data is often met by several constraints. Firstly, high within- and between-event variances in physical and skilled parameters of performance (O’Donoghue, 2004; Gregson et al., 2010) confound the assessment of systematic changes in competitive data (Bridge et al., 2013; Waldron et al., 2013). Additionally, invasive measurements (such as arterial blood sampling, muscle biopsies and expired gases) during performances are often prohibited or impractical (Bridge et al., 2013; Davis et al., 2013) and curtail a more comprehensive assessment of the physiological and metabolic responses.

A development in recent years has seen researchers devise sport-specific simulations of actual performance from detailed analyses of movement characteristics (Currell and Jeukendrup, 2008). In principle, simulation protocols provide an ergonomic framework in which to assess both the internal load of competitive performances, and the impact of specific interventions. This is achieved by regulating exercise intensity, yet enabling invasive measurements of the physiological demand. However, the task of simulating performance with adequate validity, particularly in sports characterised by dynamic movements and actions, remains a challenge (Wilkinson et al., 2009). Nevertheless, several sport-specific simulations do exist for team and individual sports that are not confined to replications of basic linear motions.

Despite the popularity of simulations for specific sports, including combat sports, a valid and sensitive protocol has yet to emerge for amateur boxing. Two previous attempts were based upon dated performances (e.g. 1994 Commonwealth Games; Smith et al., 2001) or boxing 
performed by novices over durations shorter than in higher level competitions (Davis et al., 2013). Moreover, previous attempts to simulate aspects of amateur boxing have not reported the reliability of the induced performance or physiological responses, rendering the reported external and internal loads as possibly spurious (Wilkinson et al., 2009). Indeed, the most recent attempt of Davis et al. (2013) to simulate competitive boxing included over twice the typical quantity of defences and changes in offensive performances across rounds for novices, which undermined the internal validity of the protocol. Additionally, the ambulatory demands of the simulation were based upon a movement defined as 'any visually identifiable vertical activity of the pelvis during stand and steps' (Davis et al., 2013), which is unlikely to reflect the external demand or physiological response to boxing-specific movements. Finally, the kinematic and kinetic properties of punches, which are recognised as important aspects of boxing performance (Smith, 2006; Smith et al., 2001), were not incorporated into the simulation.

Accordingly, the data reported by Thomson and Lamb (2016) have been utilised to develop a simulation protocol replicating the external demands of non-novice boxing (which involves three, three-minute rounds). Referred to hence forth as the 'boxing conditioning and fitness test' (BOXFIT), the protocol represents an externally valid replication of boxing contests incorporating the typical offensive and defensive movements observed in 92 competitive (videoed) contests (Thomson and Lamb, 2016), and data on boxing-specific ambulation (obtained by global positioning system (GPS) technology) from 12 outdoor sparring bouts. The report that follows represents the first attempt to evaluate the physical and physiological responses elicited by the BOXFIT simulation among an independent sample of amateur boxers. 


\section{Methods}

\subsection{Participants}

Twenty-eight male amateur boxers (mean \pm SD; age $22.4 \pm 3.5$ years, body mass $67.7 \pm 10.1$ kg, stature $171 \pm 9 \mathrm{~cm}$, years of experience $6 \pm 2$ years, previous contests $15 \pm 8$; predicted $\dot{V} \quad \mathrm{O}_{2 \max }=57 \pm 5 \mathrm{ml} \cdot \mathrm{kg}^{-1} \cdot \mathrm{min}^{-1}$; all right-handed thus adopting the 'orthodox' stance) were informed of the benefits and risks of the investigation prior to signing an institutionally approved informed consent document to participate in the study. Participants were informed of the procedures and potential risks of participation, and subsequently provided written informed consent. Institutional ethical approval for the experimental procedures was granted by the Faculty of Applied Sciences Ethics Committee.

\subsection{Experimental design}

The boxers were asked to maintain a normal training load and abstain from unaccustomed exercise in the preceding 72 hours. Participants underwent familiarisation trials (Currell and Jeukendrup, 2008) which involved two complete attempts of the BOXFIT simulation protocol separated by 60 minutes, the first of which employed shadow boxing exercise (i.e. movements performed with no contact) and the second included all its elements (i.e. punching handheld coaching pads). The boxers returned 72 hours later to perform the test simulation. Ninety-six hours before the familiarisation trial, participants performed a $20 \mathrm{~m}$ multi-stage fitness test (Ramsbottom et al., 1998) in order to provide estimates of their maximal oxygen uptake ( $\left.\begin{array}{cc}\dot{V} & \mathrm{O}_{2 \max }\end{array}\right)$ and maximal heart rate.

\subsection{Procedures}


The BOXFIT simulation (see below) comprised three rounds of three minutes' duration, with a one-minute rest between rounds (50 s seated, $10 \mathrm{~s}$ standing). The boxers wore a portable gas analyser (Cosmed, $\mathrm{K} 4 b^{2}$, Italy; mass $=450 \mathrm{~g}$ ) and a heart rate monitor (Polar, Electro Oy, Kempele, Finland) throughout, whilst wearing fabric hand-wrapping (450 cm length, $5 \mathrm{~cm}$ width; Adidas, Germany) and boxing gloves (284 g; Adidas, Germany) as required during actual competition. Following a 15-minute self-selected warm-up consisting of shadow boxing, jogging and punch bag exercise (Smith et al., 2001), the boxers performed the simulation protocol in a $6.1 \mathrm{~m}^{2}$ boxing ring (temperature $=19.0 \pm 3.4^{\circ} \mathrm{C}$; humidity $=41.3 \pm$ $8.5 \%)$.

\section{Boxing simulation protocol (BOXFIT)}

The boxers' actions during the simulation protocol were dictated by audio cue and included boxing-specific motions, offensive punches aimed towards coaching pads held by a qualified amateur boxing coach, and simulated defensive movements. During each minute of the simulation, the boxers cover $35.9 \mathrm{~m}$ at an average speed of $0.6 \mathrm{~m} \cdot \mathrm{s}^{-1}(35.9 \mathrm{~m}$ over $60 \mathrm{~s})$ and perform 26 punches (consisting of 15 individual attacks) and simulate 12 defences (Thomson et al., 2016) (Table 1). To complete the simulation, the boxer moves between a series of floor markings, placed at each corner and in the centre of the decagon. The internal radius of the decagon allowed for the average arm length of the boxers $\left(75 \pm 4 \mathrm{~cm}\right.$; where arm length $=\sum$ radiale-stylion length, acromial-radiale length, mid-stylion-dactylion length $(\mathrm{cm})$; Hawes and Martin, 2001). Beginning at any corner (marker 1; Figure 1) and maintaining a boxing stance throughout, the boxer moves forward to the target and performs an attack on handheld coaching pads (marker 2), before moving backwards feigning a defensive action (marker 3), and then left $\left(N \cdot \mathrm{min}^{-1}=10\right)$ or right $\left(N \cdot \mathrm{min}^{-1}=2\right)$ (marker 4$)$ before repeating the process. Though the boxers in the current study were right-handed, the protocol remains applicable to 
left-handed 'southpaw' boxers. That is, boxers adopting an 'orthodox' stance use the left hand as the 'jabbing' and 'lead' hand with the right serving as the 'rear' hand; 'southpaw' stances are the opposite (Thomson, Lamb and Nicholas, 2013).

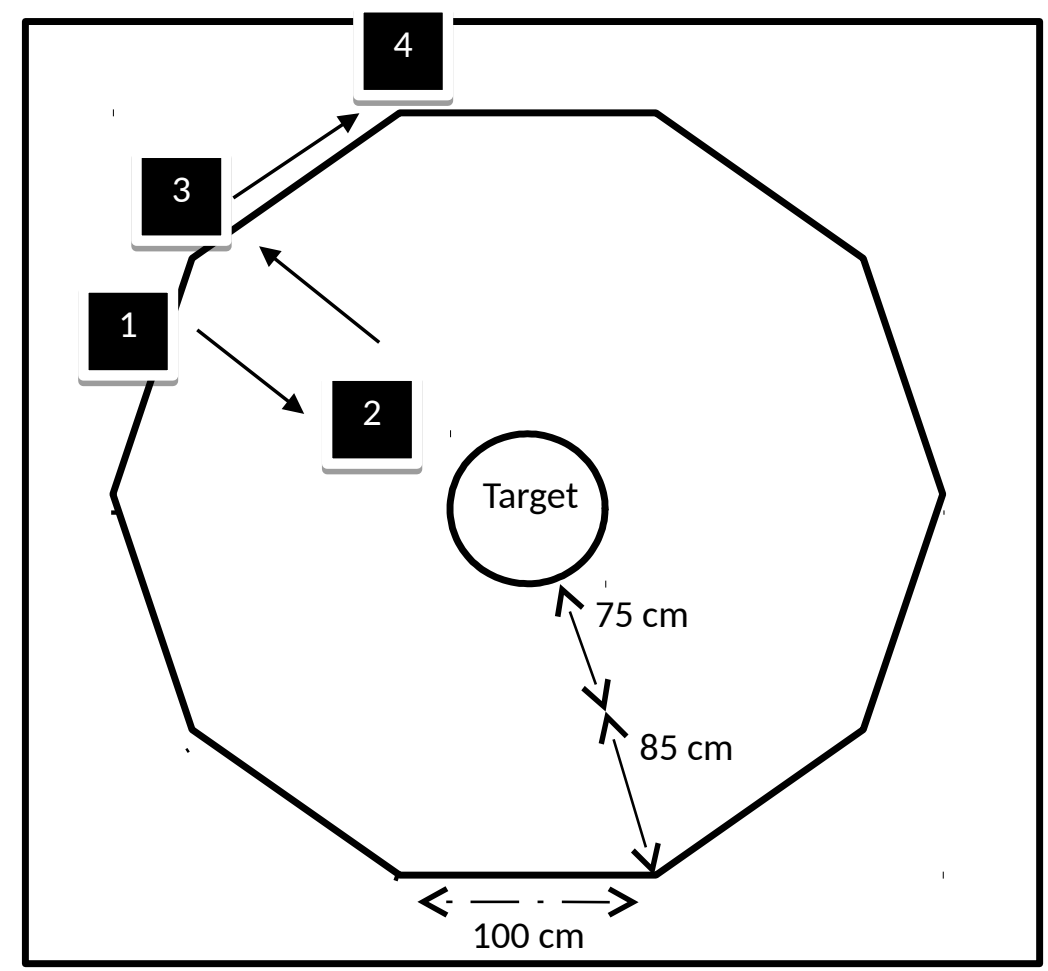

Figure 1. Schematic of the BOXFIT simulation protocol within a boxing ring (not to scale).

Table 1. The chronological order of audio cues during a one-minute of the BOXFIT.

\begin{tabular}{|c|c|c|c|c|c|c|}
\hline \multirow{2}{*}{$\begin{array}{c}\text { Direction of } \\
\text { movement }\end{array}$} & \multicolumn{4}{|c|}{ Punches to perform } & \multirow{2}{*}{$\begin{array}{c}\text { Direction of } \\
\text { movement }\end{array}$} & \multirow[t]{2}{*}{ Defence to feign } \\
\hline & $1^{\text {st }}$ & $2^{\text {nd }}$ & $3^{\text {rd }}$ & $4^{\text {th }}$ & & \\
\hline Forward & Jab & & & & Backwards & Block both arms \\
\hline \multicolumn{7}{|l|}{ Left } \\
\hline Forward & Lead hook & & & & Backward & Block right arm \\
\hline \multicolumn{7}{|l|}{ Left } \\
\hline Forward & Rear cross & Lead hook & & & Backward & Clinch \\
\hline \multicolumn{7}{|l|}{ Left } \\
\hline Forward & Jab & & & & Backward & Block both arms \\
\hline \multicolumn{7}{|l|}{ Left } \\
\hline Forward & Lead hook & & & & Backward & Block right arm \\
\hline \multicolumn{7}{|l|}{ Left } \\
\hline Forward & Rear cross & Lead hook & & & Backward & Duck \\
\hline \multicolumn{7}{|l|}{ Left } \\
\hline Forward & Rear cross & & & & Backward & Lean backwards \\
\hline Left & & & & & & \\
\hline
\end{tabular}




\begin{tabular}{|c|c|c|c|c|c|}
\hline Forward & Jab & Rear hook & & Backward & Lean backwards \\
\hline \multicolumn{6}{|l|}{ Left } \\
\hline Forward & Jab & Rear cross & Lead hook & Backward & Lean backwards \\
\hline \multicolumn{6}{|l|}{ Left } \\
\hline Forward & Rear cross & & & Backward & Foot defence \\
\hline \multicolumn{6}{|l|}{ Left } \\
\hline Forward & Jab & Rear hook & & Backward & Foot defence \\
\hline \multicolumn{6}{|l|}{ Right } \\
\hline Forward & Jab & Rear cross & Lead hook & Backward & Foot defence \\
\hline \multicolumn{6}{|l|}{ Right } \\
\hline Forward & Jab & & & Backward & \\
\hline \multirow[t]{2}{*}{ Forward } & Jab & & & & \\
\hline & Jab & Rear cross & Lead hook Rear hook & & \\
\hline Backward & & & & & \\
\hline
\end{tabular}

To provide an assessment of physical performance, wireless accelerometers (Herman Digital Trainer, USA) were attached to the wrist-region of both coaching pads (within the $10 \times 5 \mathrm{~cm}$ Velcro strap), upon which boxers delivered punches. Previous analysis of the test-retest reliability of the accelerometers in our laboratory revealed no systematic bias between repeated trials and coefficient of variations of $<5 \%$. The concurrent validity of the accelerometer was established by comparing values recorded during punching to those of a three-dimensional infrared camera system (Qualisys Track Manager, version 2.6, Qualisys Inc., Gothenburg, Sweden) (Richards, 1999). No systematic bias was observed between systems, the coefficient of determination $\left(R^{2}\right)$ was 0.72 and the standard error of the estimate using regression analysis represented $<10 \%$ of the criterion measure (Palmer and O'Connell, 2009). The sum acceleration delivered by the 78 punches to coach-held pads in each round was recorded at the end of each rounds.

\section{Physiological and metabolic measurements}

Breath-by-breath gaseous exchange measurements of oxygen uptake ( $V^{\prime}{ }_{2}$ ), carbon dioxide production ( $\dot{V} \mathrm{CO} \quad$ 2), respiratory exchange ratio (RER) and minute ventilation ( $\dot{V} \quad$ E) were recorded throughout the BOXFIT using a calibrated portable gas analyser 
(Cosmed $\mathrm{K} 4 b^{2}$, Italy). Peak and mean heart rates were recorded at a $1 \mathrm{~Hz}$ frequency throughout and subsequently expressed as raw and relative $\left(\% \mathrm{HR}_{\text {peak }}\right)$ values.

Ventilation data was used to estimate aerobic energy expenditure ( $\mathrm{EE}_{\mathrm{aer}}$; expressed in $\mathrm{kcal} \cdot \mathrm{min}^{-1}$ ) using:

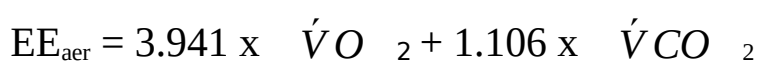

An oxygen equivalent of 3.941 was used while the non-protein respiratory quotient (npRQ) was $<1$. In the event npRQ became $>1$, an oxygen equivalent of $5.04 \mathrm{kcal} \cdot \mathrm{min}^{-1}$ was used and it was assumed all energy was derived from carbohydrate (Crisafulli et al., 2009). Given amateur boxing is known to rely substantially upon anaerobic metabolic pathways (Davis et al., 2013), an estimate of anaerobic glycolysis was also obtained by estimating excess $\mathrm{CO}_{2}$ production ( $\left.\mathrm{CO}_{2 \text { excess }}\right)$ (Crisafulli et al., 2009) as follows:

$$
\mathrm{CO}_{\text {2excess }}=\dot{V}_{\mathrm{CO}} \quad{ }_{2}-\left(\begin{array}{lll}
0.817 \mathrm{x} & \dot{V} O & 2
\end{array}\right)
$$

where 0.817 represented the resting RER (Goedecke et al., 2000).

Measures of $\mathrm{CO}_{2 \text { excess }}$ have been previously employed during intermittent exercise performance and found to correlate well with the onset of blood lactate accumulation $(r=$ 0.914, $P<0.01$ ) (Roecker et al., 2000). Capillary blood samples were collected one-minute post-exercise from the ear lobe and analysed for blood lactate using a portable device (Lactate Pro, Kyoto, Japan). Ratings of perceived exertion (RPE) were recorded upon cessation of each round using the category ratio scale (CR-10) (Foster et al., 2001) and participants were asked to provide 'global' assessments of efforts for each round.

\subsection{Statistical analysis}


Descriptive statistics (mean \pm SD) were calculated for all dependent variables and the normality of their distributions was checked using the Shapiro-Wilk test. To assess the differences in the physiological responses to the BOXFIT, along with the acceleration scores obtained during punching, repeated measures ANOVAs were employed. Where a significant $(P<0.05)$ Mauchly's test was identified, corrections to the degrees of freedom were made accordingly (O’Donoghue, 2012). Bonferroni-adjusted post-hoc $t$-tests were used to identify pairwise differences. Statistical significance was set at $P \leq 0.05$ throughout unless Bonferroni procedures were applied to a cluster of related pairwise differences. The magnitude of variance explained by main effects or interactions was quantified using partial eta squared $\left(\eta_{\mathrm{p}}^{2}\right)$ where values of 0.01 (small), 0.06 (medium) and $\geq 0.14$ (large) were used. Such values represent small (0.1), medium (0.25) and large (0.50) effects. Furthermore, for each pairwise difference, accompanying effect sizes were calculated as: $\mathrm{d}=\left(\begin{array}{llll}\dot{x} & 1^{-} & \dot{x} & 2\end{array}\right)$ / SD; where $\dot{x}_{1}$ and $\dot{x}_{2}$ represent the two sample means and SD the pooled standard deviation. Standardised effect sizes were classified as: trivial $<0.2$, small $0.2-0.6$, moderate $0.6-1.2$, large 1.2-2.0, and very large $>2.0$ (Hopkins, 2004). All data analyses were performed using either Microsoft Excel (Version 2010, Redmond, WA) or SPSS (Version 17.0; Chicago, IL).

\section{Results}

The mean heart rate response for each round and each one-minute rest period is displayed below (Figure 2). Heart rate responses were observed to vary due to round number $\left(\mathrm{F}_{2,54}=\right.$ 83.8, $\left.P<0.001, \eta_{\mathrm{p}}^{2}=0.76\right)$ representing $86-90 \%$ of $\% \mathrm{HR}_{\text {peak }}$, with values increasing significantly from one round to the next $(P<0.001$, ES $=0.62,0.90$ and 0.34 for R1 vs. R2, R1 vs. R3 and R2 vs. R3, respectively). During the rest periods, round number again exerted a significant main effect $\left(\mathrm{F}_{2,54}=14.4, P<0.001, \mathrm{\eta}_{\mathrm{p}}{ }^{2}=0.35\right)$, albeit pairwise comparisons now 
revealed significant increases from rest 1 to $2(P<0.001, E S=0.92), 1$ to $3(P<0.001 ; E S=$ 0.74), but not rest 2 to $3(P>0.05 ; E S=0.05)$.

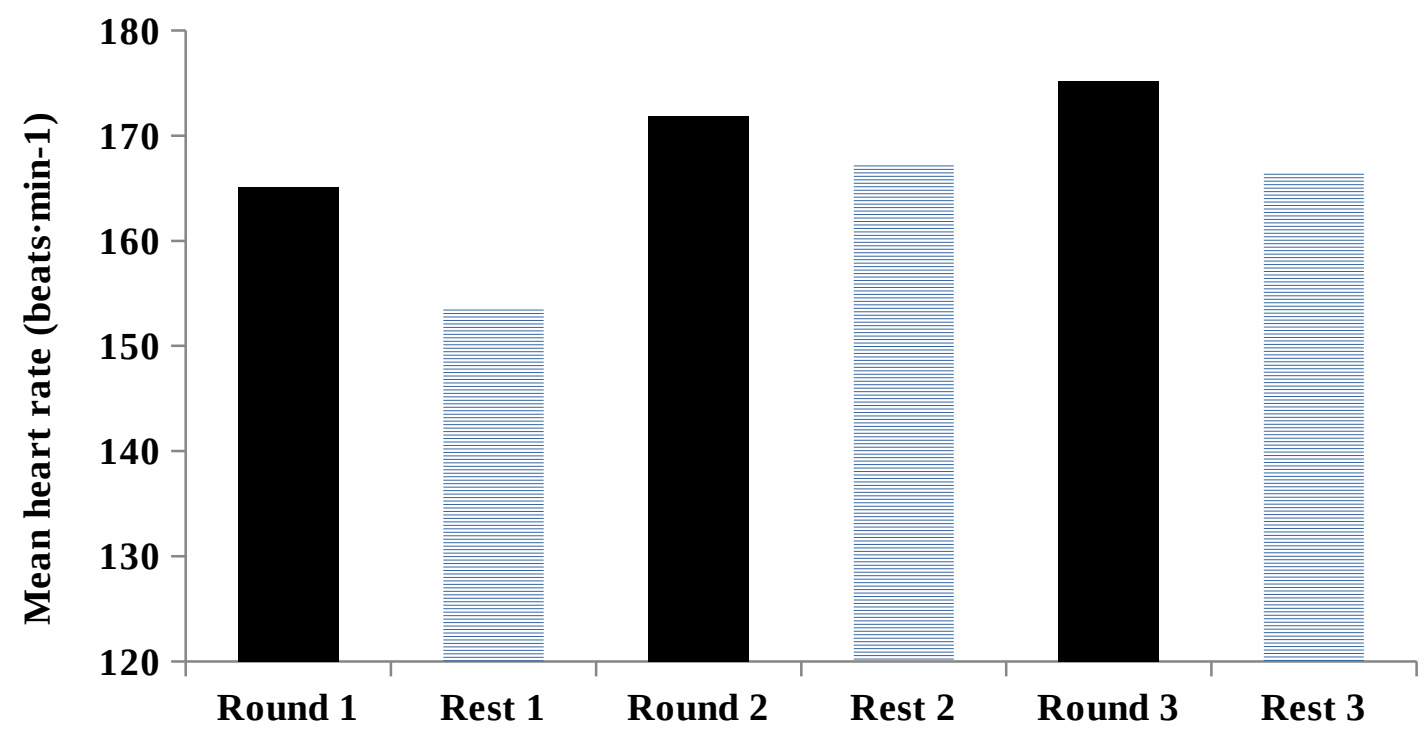

Figure 2. Mean heart rate during the BOXFIT simulation across three rounds.

Peak heart rates during exercise (Figure 3 ) also varied across rounds $\left(F_{2,54}=42.3, P<0.001\right.$, $\left.\eta_{\mathrm{p}}{ }^{2}=0.61\right)$, as did the minimum values recorded during the rest periods $\left(\mathrm{F}_{2,54}=21.7, P<\right.$ 0.001, $\left.\eta_{\mathrm{p}}^{2}=0.45\right)$. Post-hoc comparisons identified increases in peak exercise heart rates between rounds 1 and $2(P<0.05 ; E S=0.73)$ and round 1 and $3(P<0.05 ; E S=0.82)$ only which represented $91-97 \%$ of $\mathrm{HR}_{\text {peak. }}$ During recovery, there were also significant increases only between rest 1 and $2(P<0.001 ; E S=0.66)$ and rest 1 and $3(P<0.001 ; E S=0.62)$ for the minimum values. 


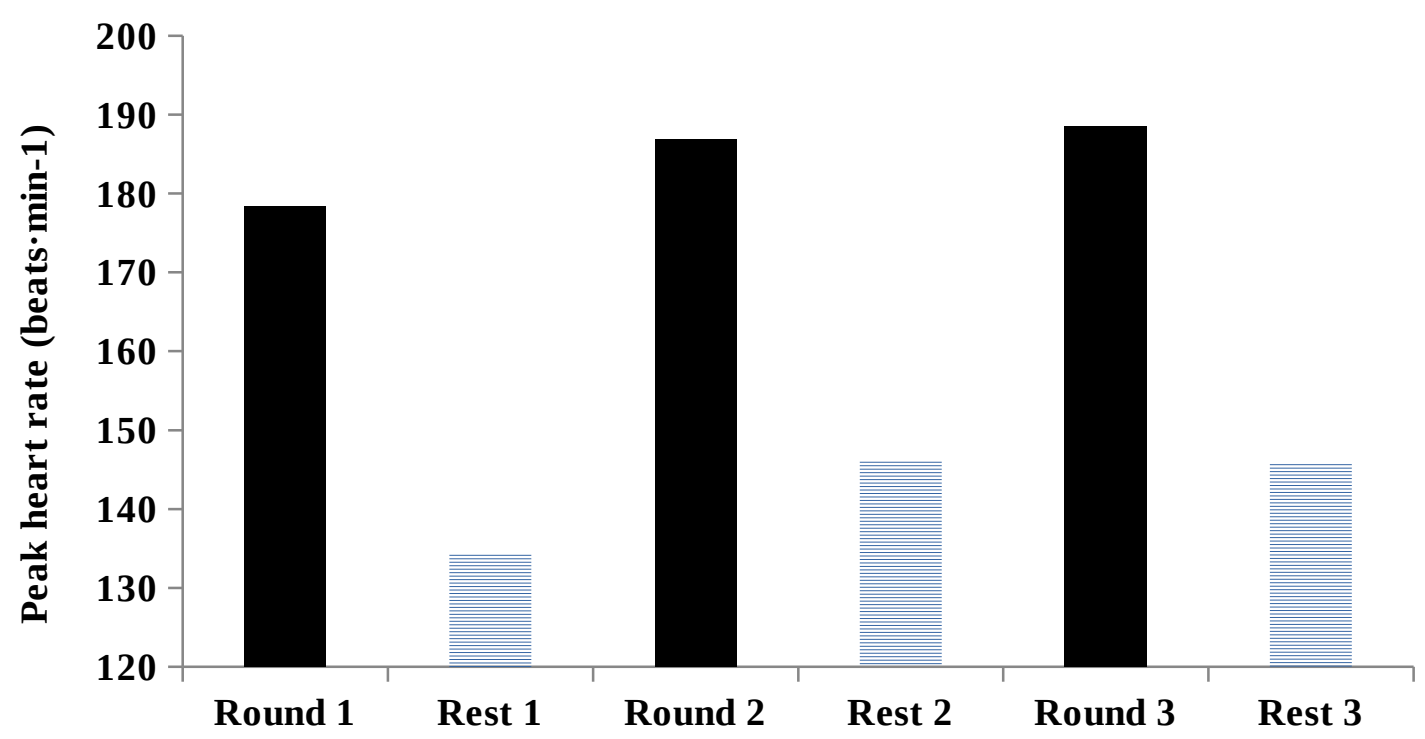

Figure 3. Peak and minimum heart rates obtained during BOXFIT exercise and rest periods across rounds, respectively.

The RPE response was significantly influenced by the round number $\left(F_{2,54}=98.3, P<0.001\right.$, $\eta_{\mathrm{p}}{ }^{2}=0.78$ ), with values increasing systematically across each round (all $P<0.05$ ) with moderate-to-large effect sizes (Round 1 vs. Round 2 ES = 0.73; Round 2 vs. Round $3 E S=$ 1.04; Round 1 vs. Round 3 ES = 1.36) (Table 2).

Table 2. RPE and $\mathrm{B}_{\mathrm{lac}}$ responses to BOXFIT performances (Mean $\pm \mathrm{SD}$ ).

\begin{tabular}{lcccc}
\hline & Round 1 & Round 2 & Round 3 & Post-simulation \\
\hline RPE & $5.8 \pm 1.4$ & $6.8 \pm 1.1$ & $8.1 \pm 1.1$ & N/A \\
\hline B $_{\text {lac }}\left(\mathbf{m m o l} \cdot \mathbf{l}^{-\mathbf{1}}\right)$ & N/A & N/A & N/A & $4.6 \pm 1.3$ \\
\hline
\end{tabular}

The absolute $\quad \dot{V} \quad \mathrm{O}_{2}$ per round (Figure 4) varied across the three rounds $\left(\mathrm{F}_{2,54}=5.3, P<\right.$ $\left.0.01, \eta_{\mathrm{p}}{ }^{2}=0.17\right)$, with values being significantly higher in R2 than R3 $(P<0.05, E S=0.44)$. Interestingly, the same measure did not vary across the rest periods $\left(\mathrm{F}_{2,54}=2.4, P>0.05, \mathrm{\eta}_{\mathrm{p}}{ }^{2}=\right.$ 
$0.08)$ and the associated effects sizes were deemed trivial-to-small $(E S=0.18-0.38)$. Mean and peak $\quad \dot{V} \quad \mathrm{O}_{2}$ were $\approx 42$ and $\approx 55 \mathrm{ml} \cdot \mathrm{kg} \cdot \mathrm{min}^{-1}$ in turn and, when expressed relative to MSFT-predicted $\quad \dot{V} \quad \mathrm{O}_{2 \max }$ values, represented $\approx 69$ and $\approx 92 \%$, respectively.

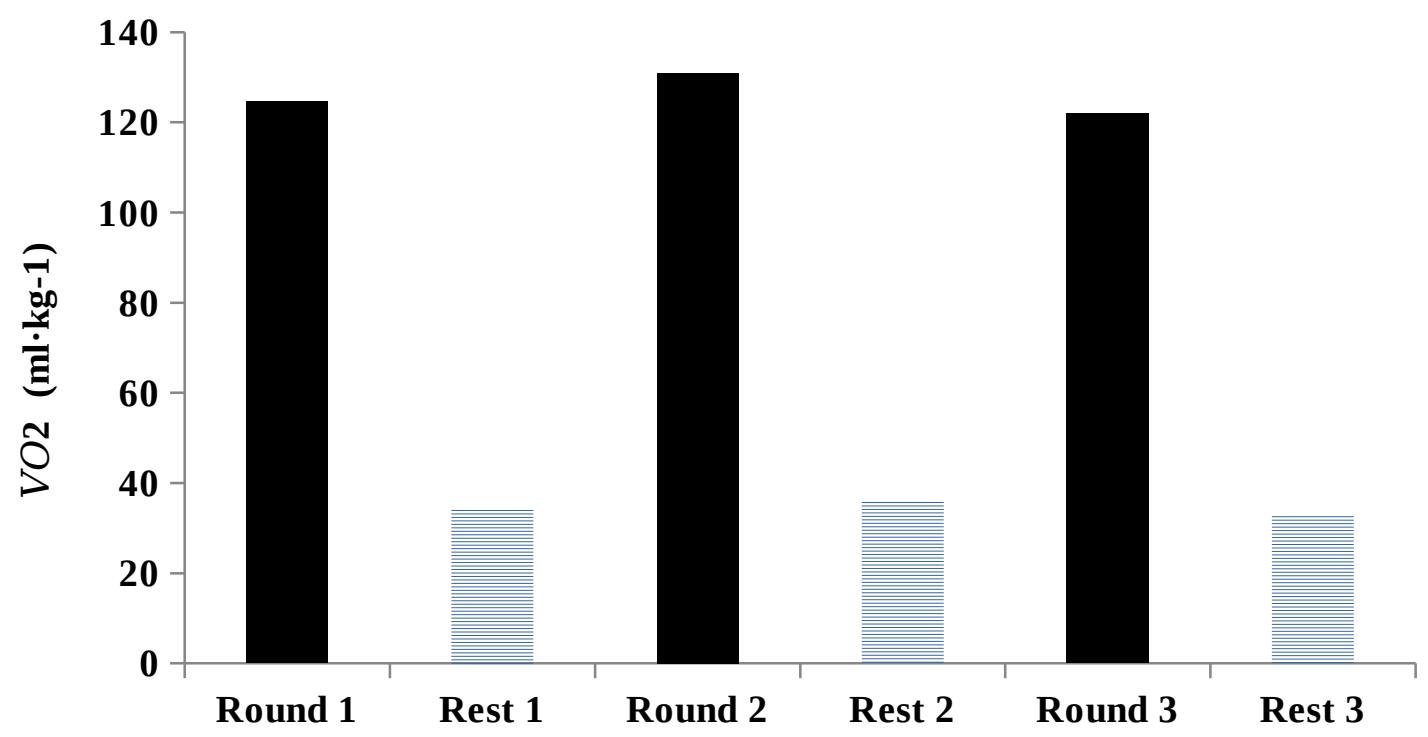

Figure 4. Absolute $\quad \dot{V} \quad \mathrm{O}_{2}$ during BOXFIT exercise and rest periods across rounds.

The $\mathrm{EE}_{\mathrm{aer}}$ of the boxers (Figure 5) was significantly influenced by the round number $\left(\mathrm{F}_{2,54}=\right.$ 4.7, $P<0.001, \eta_{\mathrm{p}}^{2}=0.15$ ), increasing positively as the simulation progressed. Post-hoc comparisons identified significant increases between the first and second $(P<0.05)$, and the first and third rounds $(P<0.05)$, albeit the accompanying effect sizes were both deemed trivial ( $E S=0.14$ and 0.17 for each comparison respectively). During the rest periods, $\mathrm{EE}_{\text {aer }}$ did not vary significantly across the rounds $\left(\mathrm{F}_{2,54}=1.7, P>0.05, \mathrm{\eta}_{\mathrm{p}}{ }^{2}=0.06\right)$. 


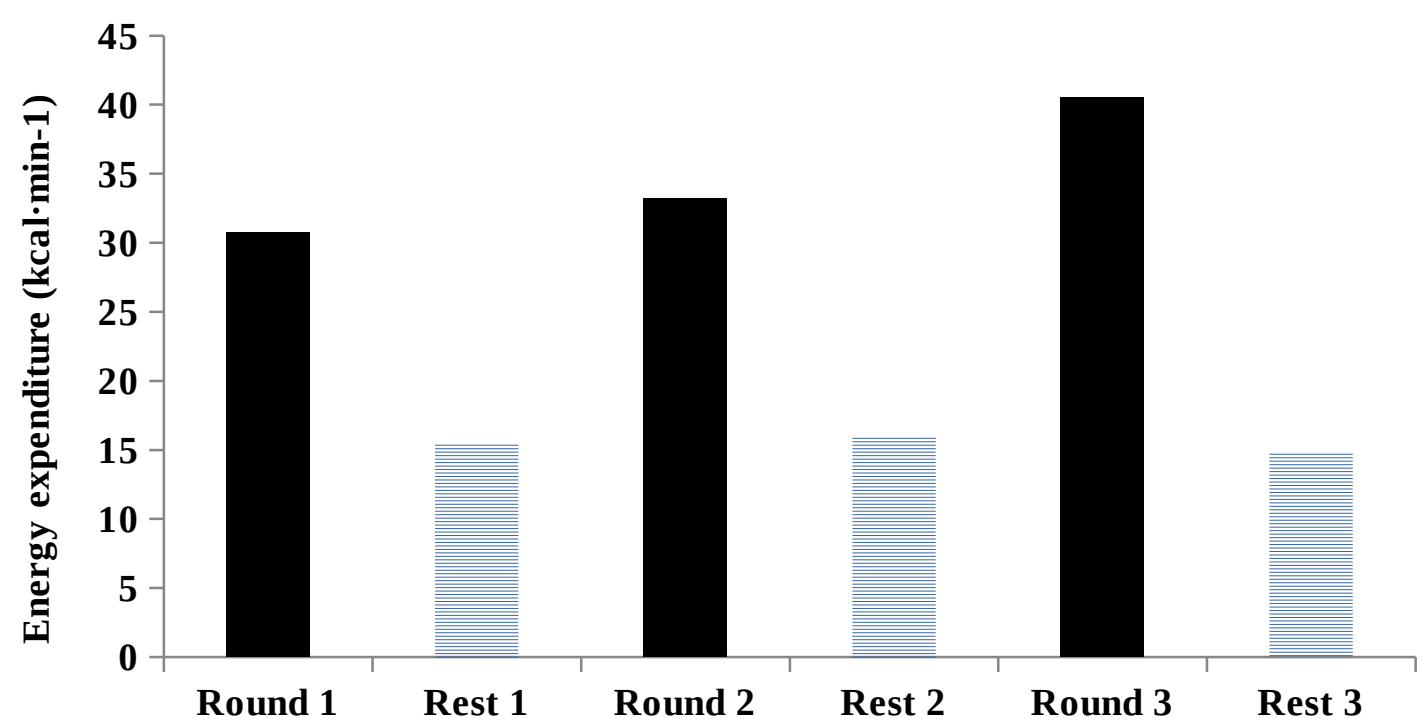

Figure 5. Mean energy expenditure $\left(\mathrm{kcal} \cdot \mathrm{min}^{-1}\right)$ during the various periods of the simulation.

Figure 6 depicts a significant effect of round on the $\mathrm{CO}_{2 \text { excess }}$ response during the exercise component of the BOXFIT $\left(\mathrm{F}_{2,54}=19.0, P<0.001, \mathrm{\eta}_{\mathrm{p}}{ }^{2}=0.41\right)$. Post-hoc analyses identified a significant increase from the first to second round $(P<0.001, E S=0.44)$, where it remained elevated during the final round $(P<0.001, E S=0.53)$, though no different to the second round $(P>0.05, E S=0.11)$. Levels of $\mathrm{CO}_{2 \text { excess }}$ during the rest periods were stable across the simulation $\left(\mathrm{F}_{2,54}=0.1, P>0.05, \eta_{\mathrm{p}}^{2}=0.00 ; E S=0.01-0.05\right)$.

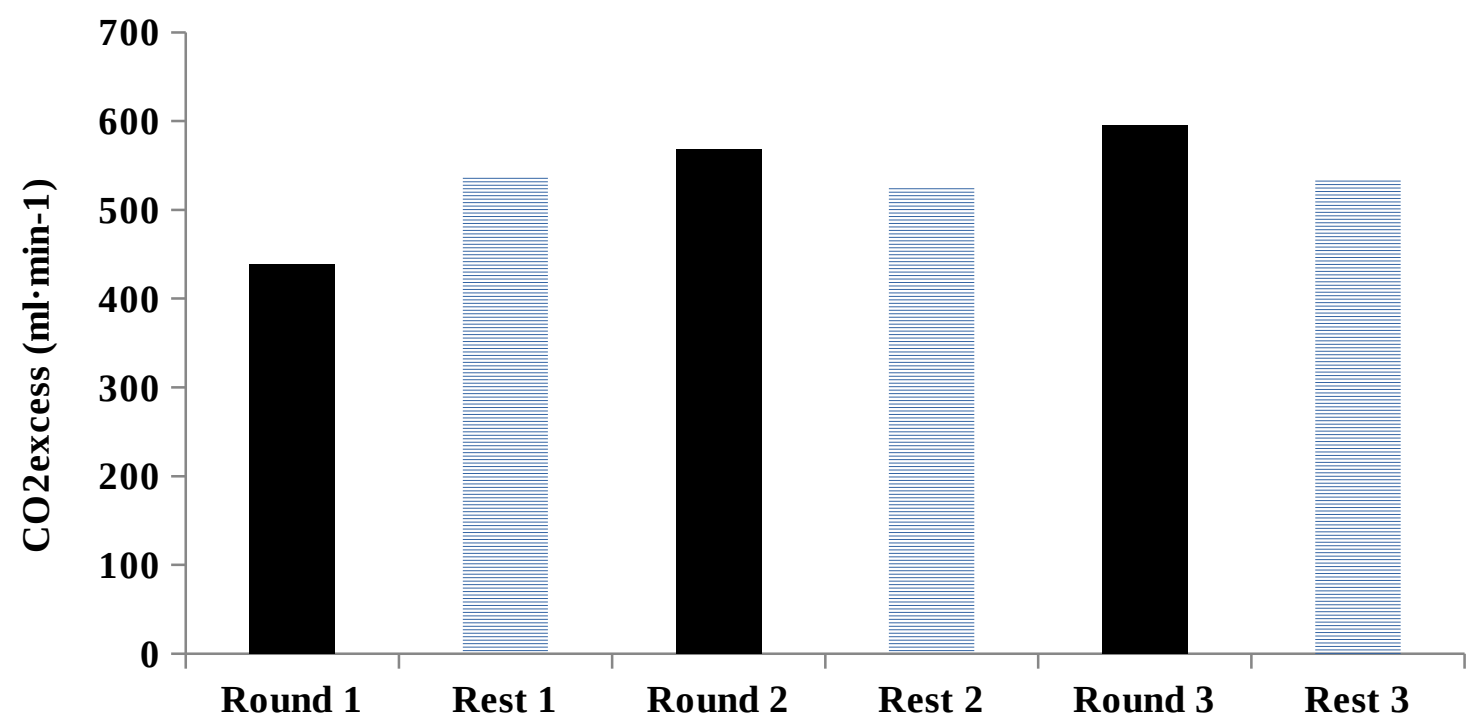

Figure 6. Mean $\mathrm{CO}_{2 \text { excess }}$ during the various periods of the simulation. 
The acceleration produced by the boxers during the punching component of the BOXFIT (Figure 7) varied significantly across rounds $\left(\mathrm{F}_{2,54}=12.2, P<0.001, \eta_{\mathrm{p}}{ }^{2}=0.31\right)$. Specifically, this reflected significant increases from round one to two $(P<0.05 ; E S=0.56)$ and round one to three $(P<0.05 ; E S=0.68)$, but not between rounds two and three $(P<0.05 ; E S=0.13)$.

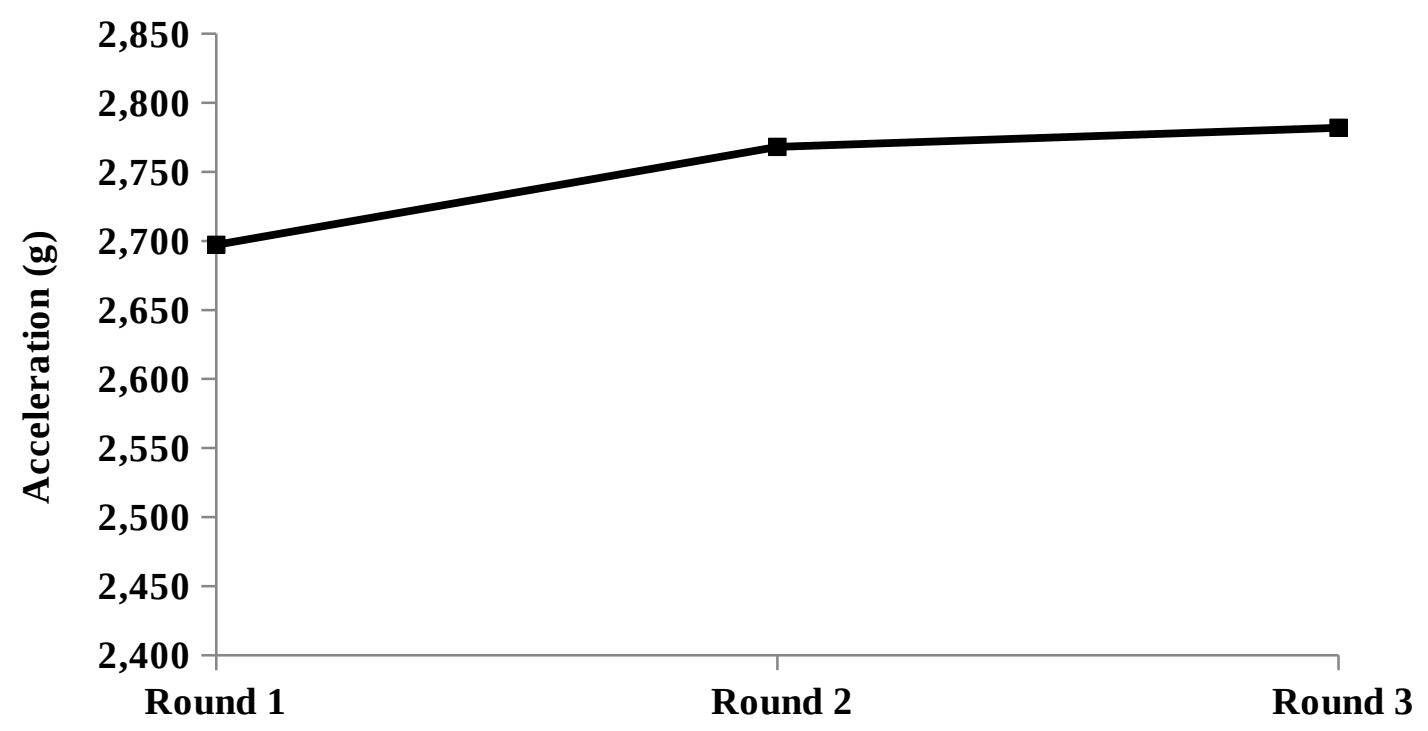

Figure 7. Total punch acceleration (mean \pm SD) delivered during the BOXFIT simulation by round.

\section{Discussion}

Utilising data gathered during an externally valid simulation protocol (BOXFIT) this study has provided the best attempt to-date to characterise the physiological and performance responses to amateur boxing. In doing so, the general findings serve to reinforce the notion that amateur boxing places a high physiological demand (internal load) upon boxers (Chaabène et al., 2015), accommodated predominantly by aerobic energy provision (Davis et al., 2013), which is seen to increase over the course of the simulation. Such knowledge will inform both conditioning practices during training and the approach to competition in boxers progressing from six to nine minute contests. 
Previous research has purported amateur boxing to be a high-intensity sport, though this supposition was largely based upon measurements of heart rate, blood lactate and RPE (Ghosh, 2010; Smith, 2006), or measurement techniques that have lacked validity (Arsenau et al., 2011). Whilst subsequent research used more invasive measures of internal (physiological) load (Davis et al., 2013), it too lacked internal validity because the exercise intensity was not regulated (despite the intention to do so), it failed to quantify any aspect related to punching performance, and did not characterise the physiological responses to a bout of three rounds of three minutes. The present study has achieved this and revealed typical mean and peak heart rates in excess of 165 and $178 \mathrm{~b} \cdot \mathrm{min}^{-1}$, and $\quad V^{\prime} \quad \mathrm{O}_{2}$ and $\mathrm{EE}_{\text {aer }}>$ $124.6 \mathrm{ml} \cdot \mathrm{kg}^{-1}$ and $>30.7 \mathrm{kcal} \cdot \mathrm{min}^{-1}$ during each round, respectively. That the mean blood lactate $\left(4.6 \mathrm{mmol} \cdot \mathrm{l}^{-1}\right)$ and $\mathrm{CO}_{\text {2excess }}\left(438.7 \mathrm{ml} \cdot \mathrm{min}^{-1}\right)$ were higher than typical resting values also reflects a contribution to energy yield from anaerobic lactacid sources. Moreover, the levels of perceived exertion recorded (increasing across rounds) are commensurate with a very high demand, and together, the data provide solid evidence that amateur boxing is indeed a high-intensity sport that requires both aerobic and anaerobic conditioning (Arsenau et al., 2011; Davis et al., 2013; Ghosh, 2010; Smith, 2006).

The 3 x 2-minute simulation protocol of Davis et al. (2013) yielded lower peak heart rates (166, 173 and $174 \mathrm{~b} \cdot \mathrm{min}^{-1}$ across rounds) than the current study $\left(178,187\right.$ and $\left.189 \mathrm{~b} \cdot \mathrm{min}^{-1}\right)$. Whilst the relative intensity of the BOXFIT is potentially higher, it is also plausible that its longer rounds (three minutes) explain this difference. The heart rate responses were also higher than those recorded during taekwondo (Campos et al., 2012), Muay Thai boxing (Crisafulli et al., 2009), karate (Doria et al., 2009), and judo (Sbriccoli et al., 2007) simulations, suggesting amateur boxing presents a higher cardiovascular strain. For the same 
reasons, the energetic profile of the BOXFIT performance also indicates a higher aerobic demand than provided by previous combat sport simulations. Whilst the mean total $\dot{V} \quad \mathrm{O}_{2}$ $\left(482 \mathrm{ml} \cdot \mathrm{kg}^{-1}\right)$ and mean total $\mathrm{EE}_{\mathrm{aer}}(360 \mathrm{kcal})$ are markedly higher than those recorded during the protocol of Davis et al. (2013) (353 $\mathrm{ml} \cdot \mathrm{kg}^{-1}$ and $146 \mathrm{kcal}$, respectively), the present study did not consider the energy derived from non-aerobic sources, and thus the $\mathrm{EE}_{\text {aer }}$ likely underestimates the true energy cost of BOXFIT performance. Indeed, Davis et al. (2013) estimated the non-aerobic energy contribution to be as much as $23 \%$, which would add considerably to the total energy cost. Despite this omission, the BOXFIT did yield a significant contribution from anaerobic metabolism given the recorded $\mathrm{CO}_{2 \text { excess }}$ values and raised lactate levels. Apart from the rest period following round one, $\mathrm{CO}_{\text {2excess }}$ was consistently higher than the values reported for Muay Thai boxing (Crisafulli et al., 2009) and probably reflects the high-intensity acyclic efforts (e.g. punching; Davis et al., 2013) being maintained by anaerobic metabolism. Notwithstanding the technical proficiency necessary for successful amateur boxing performance (Thomson and Lamb, 2016), it is therefore clear that boxers possess both high aerobic and anaerobic capacities.

Significant increases were observed between rounds in the markers of internal load. Whilst it is likely aerobic energy sources predominate throughout all nine minutes of boxing (Davis et al., 2013), it is plausible that phosphocreatine degradation and anaerobic glycolysis contributed to the earlier stages of energy yield, before an increased reliance upon aerobic sources in rounds two and three occurred (Bogdanis et al., 1996). The intermittent highintensity actions, alongside the brief recovery periods between rounds, might also have led to an excess post-exercise Oxygen (EPOC) uptake (Borsheim et al., 2003), progressively increasing across rounds. As high-intensity exercise contributes predominantly to the rapid component of EPOC, the increased $\quad \dot{V} \quad \mathrm{O}_{2}$ across rounds was likely a consequence of the 
mechanisms associated with EPOC, such as the replenishment of $\mathrm{O}_{2}$ stores in blood and muscle, resynthesis of adenosine triphosphate and creatine phosphate, lactate removal, increased body temperature, ventilation and circulation (Borsheim et al., 2003).

The increased physiological response might, however, be explained by the concomitant increase that occurred in the punch acceleration delivered by the boxers. Despite attempts to ensure a consistent demand across rounds (i.e. number of punches, defences and distance covered) it is possible that the boxers adopted a pacing strategy to avoid fatigue and optimise performance (Abbiss and Laursen, 2008) by manipulating the effort of their punches (Hall and Lane, 2001). That the recorded maximum RPE values were approximately 8 in round three suggests BOXFIT performance did not, in fact, reflect an anticipatory pacing strategy (Tucker, 2009) given maximal values were not obtained. Nevertheless, the elevated physiological load across rounds is consistent with previous attempts to simulate combat sports (Campos et al., 2012; Crisafulli et al., 2009; Davis et al., 2013).

Given the established variance in the offensive and defensive demands of amateur boxing performance according to situational variables (Thomson and Lamb, 2016), alongside the likely influence boxing 'styles' have in further modifying the demands, recognizing the presented data reflects the 'typical' demands is imperative. That is, the external demand of the BOXFIT might deviate substantially from those experienced within particular contests, even for a relatively homogenous group of boxers in terms of the weight, ability, and contest outcome (Thomson and Lamb, 2016) and so the consequent physiological response is likely to under- or over-estimate the internal load experienced during real contests. The authors are cognisant that if the BOXFIT was used as part of a boxer's preparatory training it might not provide a training stimulus of sufficient magnitude if the actual demands of a contest do 
exceed those associated with the simulation protocol. Moreover, even if the external load did accurately reflect those of a contest, the heightened psychophysiological response of boxers during competitive bouts compared to sparring (Obminski et al., 1993) suggests the BOXFIT might also provide a lowered stress response and so, the consequent physiological response might not be as high (Moreira et al., 2012). Indeed, Smith (2006) recorded post-contest $(\approx 4$ minutes) blood lactate values of $12.8 \pm 3 \mathrm{mmol} \cdot \mathrm{l}^{-1}$, far exceeding those reported in the current study, albeit among elite international amateur boxers whose external demands during bouts probably exceeded those of the BOXFIT.

It might therefore be appropriate for practitioners or coaches to modify the demands of the BOXFIT to reflect better the anticipated external and internal loads of competitive bouts. Indeed, appraising the physiological demands of tailored BOXFIT simulations according to situational influences represents a fruitful area for future research such that the range of physiological responses boxers experience could be established; this could enhance the specificity of physiological assessments and training if it were used as part of a boxer's preparatory conditioning. Nevertheless, conclusions purporting the importance of wellconditioned aerobic and anaerobic (both glycolytic and adenosine triphosphatephosphocreatine) energy systems remain relevant given athletes ought to prepare for the highest metabolic demand they might experience within competition (Dobson et al., 2007).

In replicating the external demands of amateur boxing with improved validity over previous attempts, it has been established that performance necessitates a well-developed aerobic capacity owing to the high cardiorespiratory demand. Accordingly, it is imperative that boxers employ high-intensity interval training given its ability to produce favourable adaptations (Bacon et al., 2013) in a number of variables that might facilitate successful 
boxing performance, such as an increased $\quad V^{\prime} O \quad$ 2max (permitting a higher exercise intensity throughout a contest) and improved recovery between rounds. Moreover, as the current simulation resulted in a higher cardiorespiratory demand, but lower anaerobic demand, than a previous protocol based on shorter contests ( $3 \times 2$-minute), it is suggested that boxers competing in, or transitioning to, the higher ability 3 x 3-minute format require a higher level of aerobic conditioning. Given the external validity of the technical and ambulatory movements performed during the simulation, the BOXFIT represents a method of training in itself and offers a means by which systematic changes in physiological and performancebased changes owing to intervention (e.g. hypo-hydration or energy restriction owing to body mass reduction) can be established.

\section{References}

Abbiss, C.R. and Laursen, P.B. (2008), Describing and understanding pacing strategies during athletic competition. Sports Medicine, 38, 239-252.

Arsenau, E, Mekary, S, and Leger, L.A. (2011), $\quad \dot{V} \quad \mathrm{O}_{2}$ requirements of boxing exercises. Journal of Strength and Conditioning Research, 25, 348-359.

Bacon, A.P., Carter, R.E., Ogle, E.A., and Joyner, M.J. (2013), $\quad \dot{V} \quad \mathrm{O}_{2 \max }$ trainability and high

intensity interval training in humans: a meta-analysis. PLoS ONE 8, e73182.

Bishop, D. (2008), An applied research model for the sport sciences. Sports Medicine, 38, 253-263.

Bogdanis, G.C., Nevill, M.E., Boobis, L.H., and Lakomy, H.K.A. (1996), Contribution of phosphocreatine and aerobic metabolism to energy supply during repeated sprint exercise. Journal of Applied Physiology, 80, 876-884.

Borsheim, E, and Bahr, R. (2003), Effect of exercise intensity, duration and mode on postexercise oxygen consumption. Sports Medicine, 33, 1037-1060.

Bridge, C.A., McNaughton, L.R., Close, G.L., and Drust, B. (2013), Taekwondo exercise protocols do not recreate the physiological responses of championship combat. International Journal of Sports Medicine, 34, 573-581.

Campos, F., Bertuzzi, R., Dourado, A., Santos, V., and Franchini, E. (2012), Energy demands in taekwondo athletes during combat simulation. European Journal of Applied Physiology, 112, 1221-1228.

Chaabène, H., Tabben, M., Mkaouer, B., Franchini, E., Negra, Y., Hammami, M., et al. (2015),

Amateur boxing: physical and physiological attributes. Sports Medicine, 45, 337352.

Crisafulli, A., Vitelli, S., Cappai, I., Milia, R., Tocco, F., Melis, F., et al. (2009), Physiological 
responses and energy cost during simulation of a Muay Thai boxing match. Applied Physiology, Nutrition, and Metabolism, 34, 143-150.

Currell, K., and Jeukendrup, A.E. (2008), Validity, reliability and sensitivity of measures of sporting performance. Sports Medicine, 38, 297-316.

Davis, P., Leithauser, R.M., and Beneke, R. (2013), The energetics of semi-contact 3 x 2 min amateur boxing. International Journal of Sports Physiology and Performance, 9, 233-239.

Dobson, B.P., and Keogh, J.W.L. (2007), Methodological issues for the application of timemotion analysis research. Journal of Strength and Conditioning Research, 29, 4855.

Doria, C., Veicsteinas, A., Limonta, E., Maggioni, M.A., Aschieri, P., Eusebi, F., et al. (2009), Energetics of karate (kata and kumite techniques) in top-level athletes. European Journal of Applied Physiology, 107, 603-610.

Foster, C., Florhaug, J.A., Franklin, J., Gottschall, L., Hrovatin, L.A., Parker, S., et al. (2001), A new approach to monitoring exercise training. Journal of Strength and Conditioning Research, 15, 109-115.

Ghosh, A.K. (2010), Heart rate, oxygen consumption and blood lactate responses during specific training in amateur boxing. International Journal of Applied Sports Science, 22, 1-12.

Goedecke, J.H., St Clair Gibson, A., Grobler, L., Collins, M., Noakes, T.D., and Lambert, E.V.

(2000), Determinants of the variability in respiratory exchange ratio at rest and during exercise in trained athletes. American Journal of Physiology, Endocrinology and Metabolism, 279, 1325-1334.

Gregson, W., Drust, B., Atkinson, G., and Di Salvo, V. (2010), Match-to-match variability of high-speed activities in premier league soccer. International Journal of Sports Medicine, 31, 237-242.

Hall, C.J., and Lane, A.M. (2001), Effects of rapid weight loss on mood and performance among amateur boxers. British Journal of Sports Medicine, 35, 390-395.

Hawes, M.R., and Martin, A.D. (2001), Human body composition. In Eston, R. and Reilly, T. (Eds.) Kinanthropometry and exercise physiology laboratory manual: tests procedures and data (pp. 5-46). Eastbourne, UK: Taylor and Francis.

Hopkins, W.G. (2004), How to interpret changes in an athletic performance test. Sportscience, $8,1-7$.

Moreira, A., Franchini, E., Freitas, C.G., Arruda, A.F.S., Moura, N.V., Costa, E.C., et al. (2012), Salivary cortisol and immunoglobulin A responses to simulated and official jiu-jitsu matches. Journal of Strength and Conditioning Research, 26, 2185-2191.

Obminski, Z., Stupnicki, R., Eliasz, J., Sitkowski, D., and Klukowski, K. (1993), Changes in salivary cortisol in elite boxers following laboratory exercises and fights. Biology of Sports, 10, 83-88.

O'Donoghue, P. (2004), Sources of variability in time-motion data; measurement error and within player variability in work-rate. International Journal of Performance Analysis in Sport, 4, 42-49.

O’Donoghue, P. (2012), Statistics for sport and exercise studies. London: Routledge.

Palmer, P., and O’Connell, D. (2009), Regression analysis for prediction: understanding the process. Cardiopulmonary Physical Therapy Journal, 20, 23-26. 
Ramsbottom, R., Brewer, J., and Williams, C. (1998), A progressive shuttle run test to estimate maximal oxygen uptake. British Journal of Sports Medicine, 22, 141-144.

Richards, J.G. (1999), The measurement of human motion: A comparison of commercially available systems. Human Movement Science, 18, 589-602.

Roecker, K., Mayer, F., Striegel, H., and Dickhuth, H. (2000), Increase characteristics of the cumulated excess- $\mathrm{CO}_{2}$ and the lactate concentration during exercise. International Journal of Sports Medicine, 21, 419-423.

Sbriccoli, P., Bazzuchi, I., Di Mario, A., Marzattinocci, G., and Felici, F. (2007), Assessment of maximal cardiorespiratory performance and muscle power in the Italian Olympic Judoka. Journal of Strength and Conditioning Research, 21, 738-744.

Smith, M.S. (2006), Physiological profile of senior and junior England international amateur boxers. Journal of Sports Science and Medicine, CSSI, 74-89.

Smith, M.S., Dyson, R., Hale, T., Hamilton, M., Kelly, J., and Wellington, P. (2001), The effects of restricted energy and fluid intake on simulated amateur boxing performance. International Journal of Sport Nutrition and Exercise Metabolism, $11,238-247$.

Thomson, E., and Lamb, K. (2016), The technical demands of amateur boxing: Effect of contest outcome, weight and ability. International Journal of Performance Analysis in Sport, 16, 203-215.

Thomson, E., Lamb, K., and Nicholas, C. (2013), The development of a reliable amateur boxing performance analysis template. Journal of Sports Sciences, 31(5), 516-528.

Tucker, R. (2009), The anticipatory regulation of performance: the physiological basis for pacing strategies and the development of a perception-based model for exercise performance. British Journal of Sports Medicine, 43, 392-400.

Waldron. M., Highton, J., and Twist, C. (2013), The reliability of a rugby league movement simulation protocol (RLMSP-i) designed to replicate the performance of interchanged players. International Journal of Sports Physiology and Performance, 8, 483-489.

Wilkinson, M., Leedale-Brown, D.L., and Winter, E.M. (2009), Reproducibility of physiological and performance measures from a squash-specific fitness test. International Journal of Sports Physiology and Performance, 4, 41-53. 